\title{
Comparación sensorial del cacao (Theobroma cacao L.) Nacional fino de aroma cultivado en diferentes zonas del Ecuador
}

\author{
Sensorial comparison of National fine flavor cocoa beans (Theobroma cacao L.) grown in different zones of \\ Ecuador
}

\author{
Eddyn Solórzano Chavez ${ }^{1}$, Freddy Amores Puyutaxi ${ }^{1,4}$, Juan Jiménez Barragan ${ }^{1}$, Claire Nicklin², Sonia Barzola Miranda ${ }^{3}$ \\ ${ }^{1}$ Programa Nacional de Cacao, Instituto Nacional de Investigaciones Agropecuarias (INIAP), Estación \\ Experimental Tropical Pichilingue, $\mathrm{km}$ 5, vía Quevedo-El Empalme, Apartado Postal 24. Quevedo, Los \\ Ríos, Ecuador.`eddynsch04@hotmail.com; famores.ec@gmail.com; juan.jimenez@iniap.gob.ec \\ ${ }^{2}$ Fundación Conservación y Desarrollo (C\&D), Carlos Guarderas y Gonzalo Salazar, Quito-Ecuador. clairenickin@gmail.com \\ ${ }^{3}$ Facultad de Ciencias de la Ingeniería, Universidad Técnica Estatal de Quevedo, Campus Ing. Manuel Haz \\ Álvarez, km 1.5 vía a Santo Domingo de los Tsáchilas. EC.120501. Quevedo, Ecuador. sbarzola@uteq.edu.ec \\ ${ }^{4}$ Facultad de Ciencias Agrarias, Universidad Técnica Estatal de Quevedo, Campus Ing. Manuel Haz \\ Álvarez, km 1.5 vía a Santo Domingo de los Tsáchilas. EC.120501. Quevedo, Ecuador.
}

\begin{abstract}
Resumen
$\mathrm{L}$ a presente investigación tuvo como objetivo comparar el perfil sensorial de muestras de cacao de varias zonas productoras del país. Con este propósito el Laboratorio de Calidad Integral de Cacao y Chocolate de la Estación Experimental Tropical Pichilingue del INIAP, recibió muestras de 11 asociaciones de productores. Un panel de cinco miembros con experiencia en degustación de pasta de cacao (licor) produjo datos para los siguientes componentes del sabor: cacao, acidez, amargor, astringencia, caramelo, floral, frutal, nuez y verde. El análisis comparativo de las muestras se llevó a cabo mediante la prueba no paramétrica de Kruskal \& Wallis. El grado de asociación de distintos pares de variables se examinó mediante un análisis correlacional. La relación entre muestras también se exploró mediante el análisis multivariado de componentes principales. Este análisis condujo a la identificación de las variables originales que más aportaron a la varianza de los dos primeros componentes principales. Diferencias estadísticamente significativas entre muestras se detectaron solo para el sabor a cacao. Las correlaciónes más altas se dieron entre las variables frutal y verde, frutal y astringencia, frutal y amargor, y frutal y nuez, la mayoría de signo negativo. Las variables con más aportación a la varianza $(35.20 \%)$ del primer componente principal fueron frutal, verde y amargor. Acidez, floral y nuez hicieron la mayor aportación al segundo componente principal. La aparente estructuración de dos grupos muestrales en el plano definido sugiere semejanzas y diferencias en su perfil sensorial.
\end{abstract}

Palabras clave: Pasta de cacao, perfil sensorial, diferencias de sabor.

Recibido: 30-septiembre-2014. Recibido en forma corregida: 10-noviembre-2014. Aceptado: 30-marzo-2015. Publicado como ARTÍCULO CIENTÍFICO en Ciencia y Tecnología 8(1): 37-47 Junio de 2015

\begin{abstract}
$\mathrm{T}$ he present study aimed at comparing the flavor profiles in bean samples from different cocoa growing zones of Ecuador. Samples were taken from commercial cocoa bean batches at the post harvest facilities of 11 cocoa grower associations. They sent the samples to the Chocolate and Cocoa Integral Quality Laboratory of Experimental Station Tropical Pichilingue-INIAP. Cocoa paste (liquor) obtained for each sample was tested by a five member panel to produce scores for the sensorial variables: cocoa, acidity, bitterness, astringency, sweetness, floral, frutty, nutty and raw. Kruskal-Wallis nonparametric test was applied to compare the samples. Correlation coefficients were calculated to explore the degree of closeness between different pairs of variables. Sample relationships were also examined through principal component multivariate analysis, which led to the identification of the original variables with the highest contribution to the variance in the two first principal components. Statistical differences among samples were found for cocoa flavour only. High correlation coefficients, most of negative sign, were found for frutty and raw, frutty and astringency, frutty and bitterness, and frutty and nutty. The highest contribution $(35.20 \%)$ to first principal component variance were made by: frutty, raw and bitterness. Those contributing most to the second component's total variance were: acidity, floral and nutty. The apparent structuring of two sample groups on the plane defined by the two main principal components suggests the existence of similarities and differences in their sensorial profiles.
\end{abstract}

Key words: Cocoa paste, sensory profile, differences in taste. 


\section{Introducción}

$\mathrm{E}_{\mathrm{p}}^{1}$ sabor a cacao sostiene la industria mundial de roductos de cacao y chocolates. Los granos fermentados y secos desarrollan precursores químicos que mediante la torrefacción se transforman en el sabor y aroma típico del cacao (Fowler, 2009; Pérez, 2009). La acidez, amargor y astringencia son otros sabores básicos del perfil sensorial de la pasta de cacao. El perfil puede incluir notas aromáticas complementarias como floral, frutal, nuez, malta, etc., que enriquecen las características sensoriales de los cacaos finos de aroma (Ardhana y Fleet, 2003). La intensidad e interacción de los componentes del sabor nutren las particularidades de los perfiles sensoriales de los distintos orígenes y variedades de cacao (Cros, 2004; Sukha y Butler, 2006). Cualquier esfuerzo para mejorar la comprensión del vínculo beneficio post cosecha-torrefacción-expresión sensorial, se justifica para tener más control sobre la calidad del sabor en los productos a base de cacao y chocolate. Las buenas prácticas para el control de estos procesos son importantes por su contribución al desarrollo de la expresión sensorial buscada en los productos finales para la satisfacción del consumidor.

Además del efecto de la fermentación, el perfil sensorial del cacao puede variar en función de la genética de cada árbol y la variación genética de la población de las huertas tradicionales, así como de la interacción genotipo por ambiente (Loor, 2002; Ribas, et al., 2014). Antes que una desventaja, la variación del perfil sensorial de los granos de cacao de distintos árboles, poblaciones, orígenes, y zonas productoras, es más bien un caudal de alternativas para el segmento de la industria de chocolates con alto contenido de cacao. Esta circunstancia ayuda al desarrollo de recetas de nuevos productos necesarios para impulsar sus marcas comerciales. De ahí que la búsqueda y desarrollo de orígenes de cacao más específicos es una tendencia creciente, particularmente en los mercados maduros. Los orígenes dotados de una identidad conocida y comprobable, uno de cuyos componentes es la identidad sensorial, son fuente de oportunidades para que su precio de mercado se beneficie de premios atractivos que fomenten su producción.

Son frecuentes las referencias de compradores acerca de la presencia de diferencias sensoriales entre los cacaos de países distintos y entre zonas diferentes dentro de un mismo país, tanto para el cacao de huertas tradicionales como entre variedades policlonales y monoclonales (Amores et al., 2007).

Sin embargo, la evidencia empírica producida por estudios planificados con este fin escasea. Frente a esta necesidad se condujo una investigación exploratoria para comparar el perfil sensorial del cacao de distintas zonas productoras del Ecuador, en la búsqueda de particularidades de sabor para su diferenciación. La confirmación de diferencias reales entre zonas puede convertirse en el punto de partida para desarrollar orígenes específicos, con capacidad para acceder a nichos de mercado con más valor.

\section{Materiales y métodos}

I a investigación se realizó durante el periodo 20112013, en el Laboratorio de Calidad Integral de Cacao y Chocolate del Programa Nacional de Cacao de la Estación Experimental Tropical Pichilingue del INIAP, cantón Quevedo, Provincia de Los Ríos.

A través de varios componentes del sabor se caracterizaron un grupo de muestras de cacao colectadas en los centros de beneficio post cosecha de 11 asociaciones de productores, asentadas en distintas zonas del Ecuador (Figura 1).

\section{Procedencia de las muestras de cacao}

Las áreas de influencia de cada asociación se localizan en diferentes provincias. Unión de Asociaciones Artesanales "Eloy Alfaro", Asociación de Productores de Atacames (APROCA) y Corporación de Organización Campesinas de Esmeraldas (COCPEC) en la provincia de Esmeraldas. Fortaleza del Valle en la provincia de Manabí. Asociación de Productores Orgánicos de Vinces (APOV) en la provincia de Los Ríos. Federación Nacional de Productores de Cacao del Ecuador (FEDECADE) y Asociación Buena Suerte en la provincia del Guayas. La Corporación Agrícola Cacaotera del Cantón Echeandía (CORAGRICACE) y la Asociación de productores de Las Naves (UCOCS) en la provincia de Bolívar. La Asociación Miss Ecuador en la provincia de Sucumbíos y la Asociación San Carlos en la provincia de Orellana, ambas en el nororiente de la región Amazónica (Cuadro 1).

\section{Muestreo}

El proceso de beneficio postcosecha fue realizado por cada asociación de acuerdo a sus parámetros y condiciones climáticas en cada zona; en el Cuadro 2 se detalla el proceso de fermentación y secado. A partir de lotes procesados se tomaron 3 muestras de cacao a razón de $1 \mathrm{~kg}$ por muestra. Las muestras provinieron de lotes procesados en distintos momentos del año. Una vez que llegaron al laboratorio se determinó la humedad de los granos de cada muestra. Aquellas con más contenido de humedad que el estándar (7\%), se dispusieron en un tendal para completar el secado. Todas se sometieron a una prueba de corte para estimar el grado de fermentación (Cuadro 3) donde la sumatoria 
Cuadro 1. Descripción meteorológica de las zonas de muestreo

\begin{tabular}{|c|c|c|c|c|c|c|}
\hline Asociaciones & Cantón & Provincia & $\begin{array}{l}\text { Altitud }^{1} \\
(\mathrm{msnm})\end{array}$ & $\begin{array}{l}\begin{array}{l}\text { Heliofanía } \\
\text { (horas/año) }\end{array} \\
\end{array}$ & $\begin{array}{c}\text { Precipitación }^{1} \\
(\mathbf{m m} / \mathbf{a n n o})\end{array}$ & $\begin{array}{c}\text { Humedad relativa }{ }^{1} \\
(\%)\end{array}$ \\
\hline Eloy Alfaro & Eloy Alfaro & Esmeraldas & 55 & - & 3681.10 & 85 \\
\hline Aproca & Atacames & Esmeraldas & 5 & 1009.40 & 2646.20 & 82 \\
\hline Cocpe & Quinindé & Esmeraldas & 115 & - & - & - \\
\hline Fortaleza del Valle & Calceta & Manabí & 15 & 1128.20 & 1615.50 & 83 \\
\hline Buena Suerte & El Empalme & Guayas & 28 & - & - & - \\
\hline Apov & Vinces & Los Ríos & 50 & 801.20 & 2059.70 & 81 \\
\hline Fedecade & Naranjal & Guayas & 14 & - & 667.50 & 91 \\
\hline San Carlos & San Carlos & Orellana & 345 & 1031.50 & 4143.90 & 91 \\
\hline Miss Ecuador & Miss Ecuador & Sucumbíos & 580 & - & - & - \\
\hline Coragricace & Echeandía & Bolívar & 308 & - & - & - \\
\hline Ucocs & Las Naves & Bolívar & 679 & - & - & - \\
\hline
\end{tabular}

${ }^{1}$ Instituto Nacional de Meteorología e Hidrología (2012)

de la almendras con alta y mediana fermentación es el total del porcentaje de la muestra, de acuerdo a la norma INEN 176. Esta información, fue de utilidad al momento de la interpretación de los resultados sensoriales. Cada muestra se colocó en sacos de malla debidamente codificados, antes de proceder a su almacenamiento en un cuarto con temperatura controlada de $20^{\circ} \mathrm{C}$ y dotado de un deshumidificador para controlar la humedad ambiental alrededor de $50 \%$. Las muestras permanecieron en el cuarto hasta el momento del tostado para obtener la pasta.

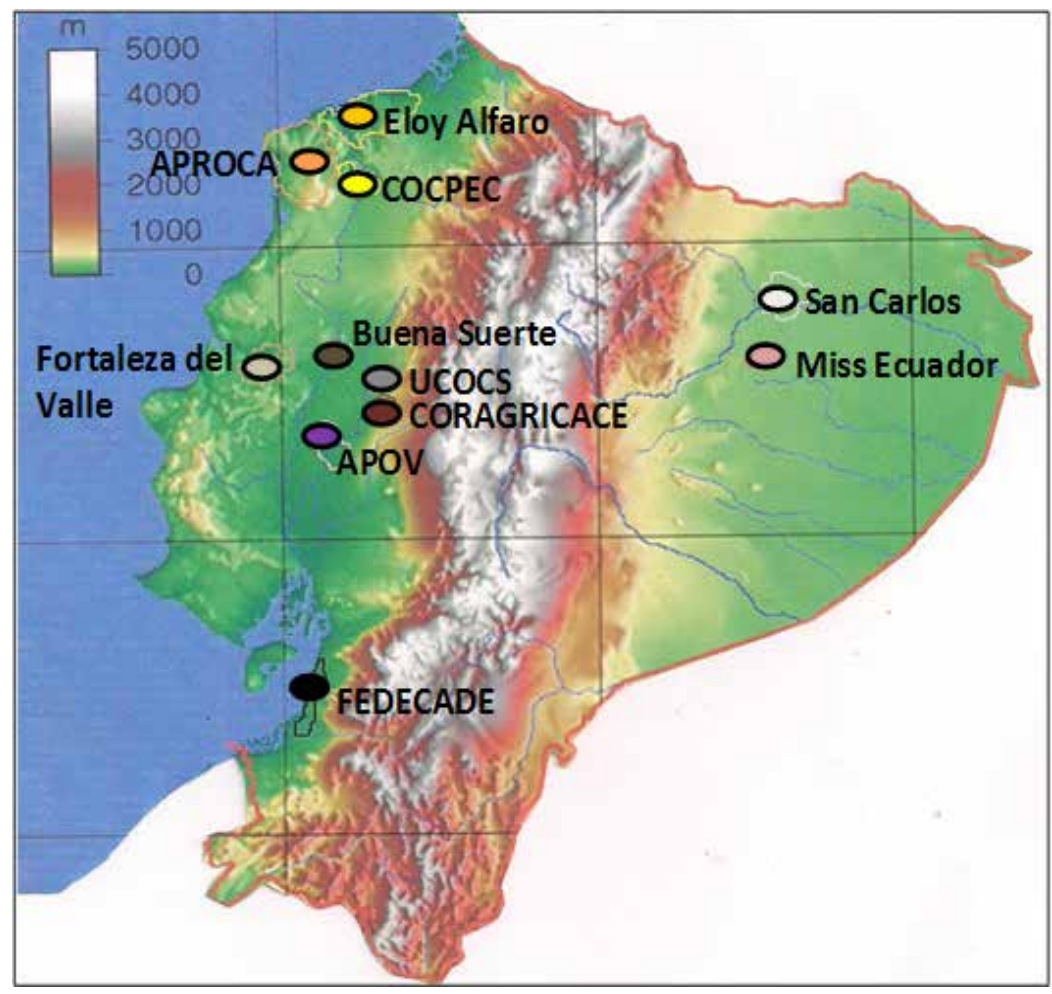

Figura 1. Ubicación geográfica de los sitios de recolección de muestras 
Cuadro 2. Proceso de postcosecha (fermentación y secado)

\begin{tabular}{|c|c|c|c|c|}
\hline Asociaciones & Tipo de Fermentación & $\begin{array}{c}\text { Tiempo de } \\
\text { fermentación } \\
\text { (días) }\end{array}$ & Tipo de secado & $\begin{array}{l}\text { Tiempo de secado } \\
\text { (días) }\end{array}$ \\
\hline Eloy Alfaro & Cajones de madera & 4 & Secado natural marquesina & 5 \\
\hline APROCA & Cajones de madera & 4 & Secado natural marquesina & 5 \\
\hline COCPE & Cajones de madera & 4 & Secado natural marquesina & 5 \\
\hline Fortaleza del Valle & Cajones de madera & 4 & Secado natural marquesina & 5 \\
\hline Buena Suerte & Cajones de madera & 4 & $\begin{array}{l}\text { Secado natural marquesina y } \\
\text { artificial secadora a gas }\end{array}$ & 5 \\
\hline UCOCS & Cajones de madera & 4 & $\begin{array}{l}\text { Secado natural marquesina y } \\
\text { artificial secadora a gas }\end{array}$ & 5 \\
\hline APOV & Cajones de madera & 3 & $\begin{array}{l}\text { Secado natural marquesina y } \\
\text { artificial secadora a gas }\end{array}$ & 7 \\
\hline FEDECADE & Montón tipo lagarto & 2 & $\begin{array}{l}\text { Secado natural tendal de } \\
\text { cemento }\end{array}$ & 4 \\
\hline San Carlos & Cajones de madera & 4 & $\begin{array}{l}\text { Secado natural tendal de } \\
\text { cemento }\end{array}$ & 5 \\
\hline Miss Ecuador & Cajones de madera & 4 & $\begin{array}{l}\text { Secado natural tendal de } \\
\text { cemento }\end{array}$ & 5 \\
\hline CORAGRICACE & Cajones de madera & 5 & $\begin{array}{l}\text { Secado natural tendal de ce- } \\
\text { mento y tendal de madera }\end{array}$ & 6 \\
\hline
\end{tabular}

Cuadro 3. Porcentajes de fermentación y defectos de los granos de las muestras de las zonas productoras

\begin{tabular}{lccccc}
\hline \multirow{2}{*}{ Asociación } & \multicolumn{3}{c}{ Fermentación (\% almendras) } & \multicolumn{2}{c}{ Defectos (\% almendras) } \\
\cline { 2 - 6 } & Alta & Mediana & Total & Violeta & Pizarra \\
\hline Eloy Alfaro & 58 & 38 & 96 & 2 & 2 \\
APROCA & 55 & 35 & 90 & 10 & 0 \\
COCPE & 38 & 42 & 80 & 9 & 11 \\
Fortaleza del Valle & 63 & 31 & 94 & 6 & 0 \\
Buena Suerte & 51 & 36 & 87 & 13 & 0 \\
UCOCS & 32 & 41 & 73 & 9 & 18 \\
APOV & 45 & 34 & 79 & 19 & 2 \\
FEDECADE & 33 & 20 & 53 & 40 & 7 \\
San Carlos & 48 & 42 & 90 & 10 & 0 \\
Miss Ecuador & 37 & 41 & 78 & 22 & 0 \\
CORAGRICACE & 45 & 46 & 91 & 9 & 0 \\
\hline Promedio & 46 & 37 & 83 & 14 & 4 \\
\hline
\end{tabular}

\section{Elaboración de pasta de cacao}

De cada muestra se procesaron 200 gramos de cacao para obtener la pasta. La torrefacción o tostado de las almendras se llevó a cabo en una estufa Mermmet UF con aire forzado.

Previamente se realizaron ejercicios con distintas temperaturas y tiempos para decidir la temperatura de tostado, en función del tamaño del grano y porcentaje de cascarilla. Con esto se buscó reducir el riesgo de tostado excesivo o falta de tostado de los granos que causa defectos en el sabor (Barel et al., 1985). La combinación de esta información con la experiencia 
de otros estudios permitió la selección del régimen de tostado aplicado a cada muestra.

La combinación de la temperatura y tiempo de tostado se describe a continuación. Las muestras de Eloy Alfaro, APROCA, COCPEC se tostaron a una temperatura de $110^{\circ} \mathrm{C}$ porque el cacao de esta zona es más acriollado o en su mayoría corresponde a la variedad Criollo (Zyzelewicz et al., 2014; Sacchetti et al., 2015). Para las muestras de Fortaleza del Valle la temperatura de tostado fue de $112^{\circ} \mathrm{C}$. El cacao de la provincia de Manabí representa a la variedad Nacional típica de Ecuador. En las asociaciones de Buena Suerte, FEDECADE, APOV, CORAGRICACE, UCOCS, Miss Ecuador y San Carlos se encuentra el complejo Nacional derivado de los cruzamientos naturales entre Nacional, Trinitario y otras variedades del tipo Criollo y Forastero; en este caso, las almendras se tostaron a $115^{\circ} \mathrm{C}$. En todos los casos el tiempo de tostado fue de 12 minutos (Solórzano, 2011; Oracz y Nebesny, 2014).

Al completar el tostado los granos se dejaron enfriar por varios minutos antes de proceder al descascarillado manual para separar los cotiledones de la testa o cascarilla. Los cotiledones libres de la cascarilla y otras impurezas se trituraron en una licuadora industrial que los transformó en partículas pequeñas o "nibs". El menor tamaño de las partículas facilitó su posterior transformación en pasta de cacao con un molino refinador. Cada muestra se refinó por un periodo de 30 minutos.

Una vez obtenida la pasta se colocó en moldes de plástico que se introdujeron luego en un refrigerador para su endurecimiento. Al completarse un día en el refrigerador los pequeños trozos rectangulares de pasta se extrajeron de los moldes, empacaron en papel aluminio y colocaron en bolsas de plástico membretadas para distinguir una muestra de otra.

Después se almacenaron y mantuvieron en un congelador a $4^{\circ} \mathrm{C}$. Allí permanecieron hasta el momento del análisis sensorial.

\section{Análisis sensorial}

Un panel de catadores compuesto por cinco personas con experiencia en la degustación de pasta (licor) de cacao, degustó y cuantificó una serie de parámetros sensoriales. Para la cuantificación de cada variable se aplicó la escala ordinal $0-10$, donde 0 es ausente, 1 a 2 bajo, 3 a 5 medio, 6 a 8 alto, 9 a 10 muy alto (ISO, 1988; Puerari et al., 2012). La evaluación se hizo para cada una de las tres muestras de cada zona reportándose respectivos datos y promedio por muestra por zona. Los resultados se describieron mediante la construcción de gráficos de barras. El vector en el extremo superior de cada barra representa la desviación estándar.
Para cumplir el objetivo de la caracterización sensorial se colocaron pequeños bloques de pasta de cacao en vasitos plásticos identificados con códigos numéricos distintos, cada uno de tres dígitos. De esta manera el origen de cada muestra fue totalmente desconocido para los integrantes del panel que hizo la evaluación.

Los vasitos plásticos conteniendo las muestras se colocaron en baño de maría a una temperatura entre 45 y $50^{\circ} \mathrm{C}$ para calentar y diluir la pasta. La pasta caliente facilitó la tarea de los catadores por la mayor volatilización de los compuestos aromáticos una vez que ésta se coloca en la boca. Cada miembro del panel registró en un formulario el valor de la intensidad sensorial que iba percibiendo para cada variable (ISO, 1988; Cedeño, 2010; Solórzano, 2011). Al final de cada sesión los formularios se recolectaban y digitalizaban para la construcción gradual de la respectiva base de datos. La catación de todas las muestras se completó en varias sesiones. Nunca se evaluaron más de cinco muestras por sesión. En total se cataron 33 muestras de pasta.

Se cuantificaron las siguientes variables sensoriales: cacao, floral, frutal, nuez, caramelo, acidez, amargor, astringencia y verde. A medida que la intensidad de cualquier nota sensorial iba en aumento, se cuantificaba con un valor más alto de la escala acordada. El significado de cada variable se describe a continuación:

Cacao, describe el sabor típico a granos de cacao fermentado, seco y tostado, libre de cualquier contaminación.

Floral, describe una sensación de frescura en la boca. El licor se presenta como si estuviera perfumado. Referencias son los aromas de las flores de los cítricos, jazmines, violetas y otras.

Frutal, describe sensaciones cercanas al sabor de fruta madura con una nota de aroma a dulce, ligeramente ácido y agradable al gusto.

Nuez, describe una sensación cercana al sabor de nuez o maní.

Caramelo, describe una sensación cercana al azúcar y otros compuestos como los edulcorantes sintéticos.

Acidez, describe sensaciones que se originan en la presencia de ácidos volátiles y no volátiles. Puede ser muy intensa si el nivel de fermentación es insuficiente. Amargor, se describe como una sensación fuerte relacionada con los compuestos químicos, específicamente las purinas. La fermentación insuficiente aumenta su intensidad.

Astringencia, se describe como una sensación de sequedad en la boca producto de la precipitación de las proteínas de la saliva. La fermentación insuficiente aumenta su intensidad. 
Verde, se describe como una sensación a sabor manchosocrudo, producto de la fermentación insuficiente. A esta característica también se conoce como crudo.

\section{Análisis estadístico}

La descripción gráfica de los resultados sensoriales con barras permitió un primer acercamiento en el intento de explorar las diferencias entre muestras. El análisis mediante la prueba de Kruskal y Wallis (5\%) permitió la comparación estadística entre muestras para cada variable. Con un análisis correlacional, se examinó el comportamiento de distintos pares de variables para determinar su nivel de asociación. Posteriormente, el análisis multivariado de componentes principales permitió abordar la comparación de las muestras con un enfoque más integral, examinando en el plano definido por los dos componentes principales con el mayor contenido de varianza, el patrón de distribución de los puntos con toda la información sensorial de las distintas muestras. El análisis sirvió además para identificar aquellas características con mayor aportación a la varianza contenida en el primer y segundo componente principal.

\section{Resultados y discusión}

$\mathrm{L}$ a expresión sensorial del cacao contiene un fuerte componente genético que luego del beneficio postcosecha y torrefacción se traduce en diferencias para una o más variables de sabor en muestras de distintos orígenes (Cros, 2004; Cedeño, 2010). Las diferencias sensoriales entre muestras de cacao de distintas zonas productoras del país, es una hipótesis surgida a la luz de las diferencias genéticas entre plantas individuales y poblaciones de huertas cacaoteras tradicionales (Loor, 2002; Amores et al., 2009). Las diferencia sensoriales están condicionadas por la interacción genotipo por ambiente. Las condiciones de fermentación y secado son parte de ese ambiente (Jiménez et al., 2011; Ho et al., 2015).

Una de las características sensoriales exhibió una amplia variación que se deduce de la magnitud de la desviación estándar (Figuras 2 y 3). Sin embargo, el análisis univariado o multivariado solo arrojó diferencias estadísticamente significativas para el sabor a cacao (Cuadro 4). Los coeficientes de correlación más altos se dieron para los siguientes pares de variables: frutal y verde (-0.78), frutal y astringencia (-0.77), frutal y amargor (-0.73), cacao y nuez ( 0.59$)$, y cacao y verde $(-0.59)$, solo el coeficiente entre cacao y nuez es positivo (Cuadro 5).

Los perfiles sensoriales de las muestras del presente estudio son únicos en su comportamiento individual. Sin embargo, las diferencias estadísticas encontradas solo para el sabor a cacao, confirman una gran variación en las lecturas entre muestras de cada zona para los demás componentes del perfil (acidez, astringencia, amargor, floral, frutal, etc.). Esta variación impidió la separación estadística entre muestras al analizar otras variables sensoriales. Cualquier diferencia real entre muestras para rasgos sensoriales distintos al de cacao, puede haber quedado enmascarada por esta circunstancia (Tran et al., 2015). La generación de datos sensoriales mediante la catación de pasta de chocolate es un proceso de gran subjetividad. Los sentidos del gusto y el olfato del catador son los instrumentos directamente involucrados en la medición sensorial (Briz y García,

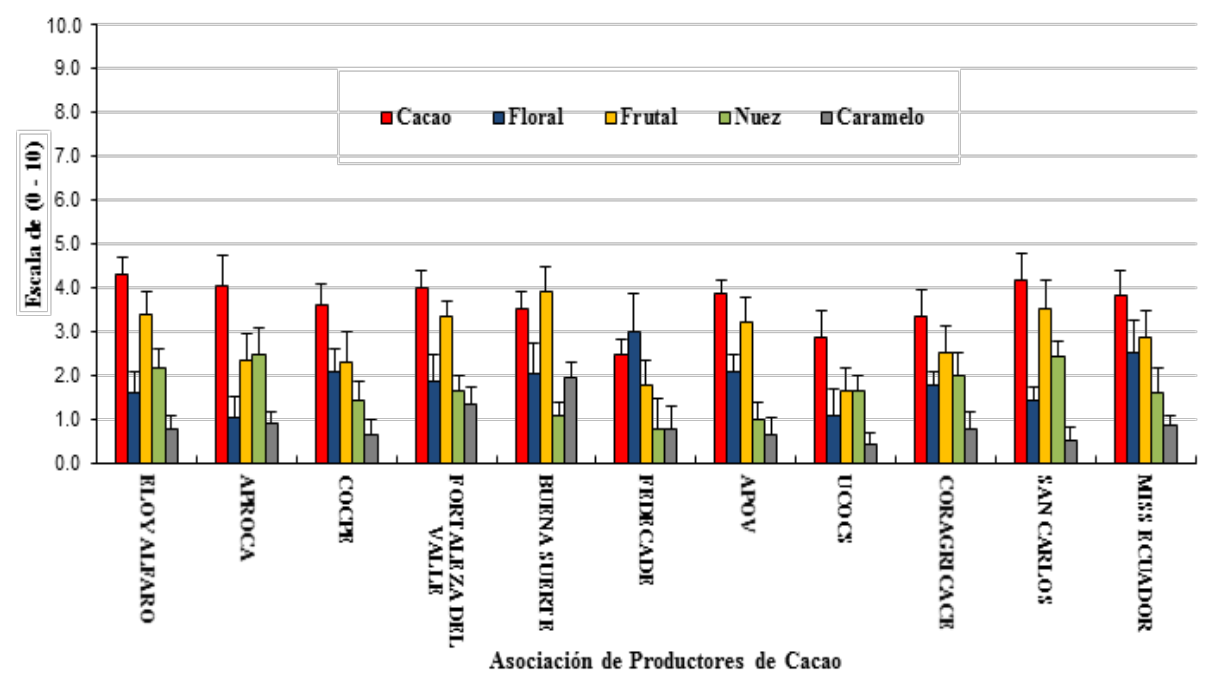

Figura 2. Comportamiento de las variables cacao, floral, frutal, nuez y acaramelado en muestras de cacao de distintas zonas productoras 


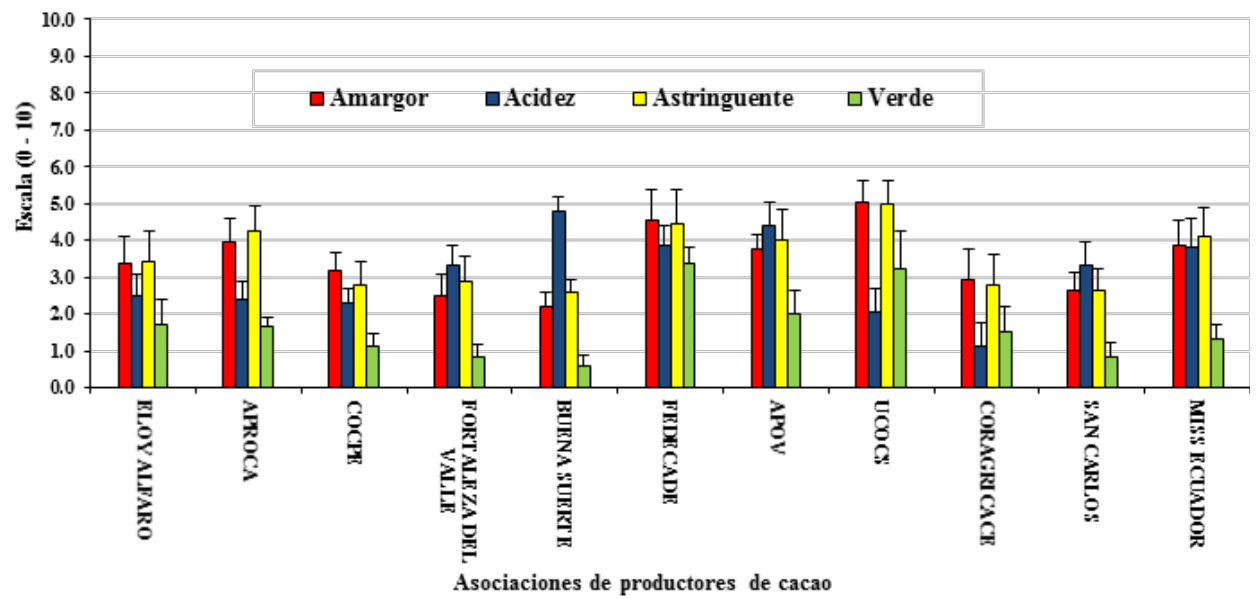

Figura 3. Comportamiento de las variables amargor, acidez, astringencia, verde, en muestras de cacao de distintas zonas productoras

Cuadro 4. Comparación de las variables sensoriales utilizadas para caracterizar muestras de cacao de distintas zonas productoras

\begin{tabular}{|c|c|c|c|c|c|c|c|c|c|}
\hline \multirow[b]{2}{*}{ Asociación } & \multicolumn{8}{|c|}{ Variables sensoriales $(0-10)$} & \multirow[b]{2}{*}{ Verde } \\
\hline & Cacao & Floral & Frutal & Nuez & Caramelo & Amargor & Acidez & Astringencia & \\
\hline Eloy Alfaro & $3.80 \mathrm{abcd}$ & 1.10 & 4.40 & 3.00 & 1.10 & 3.00 & 2.50 & 2.20 & 0.30 \\
\hline APROCA & $4.10 \mathrm{bcd}$ & 0.80 & 2.20 & 3.10 & 0.70 & 3.70 & 2.20 & 3.80 & 2.10 \\
\hline COCPE & $3.50 \mathrm{abcd}$ & 1.50 & 1.90 & 1.80 & 0.40 & 3.90 & 1.80 & 4.10 & 2.00 \\
\hline Fortaleza del Valle & $3.30 \mathrm{abcd}$ & 1.60 & 3.60 & 1.20 & 1.20 & 2.40 & 3.00 & 2.80 & 0.90 \\
\hline Buena Suerte & $3.00 \mathrm{ab}$ & 2.10 & 4.00 & 0.80 & 1.50 & 2.20 & 5.20 & 2.90 & 0.90 \\
\hline UCOCS & $2.70 \mathrm{a}$ & 0.90 & 1.60 & 1.50 & 0.70 & 4.40 & 2.20 & 4.40 & 2.70 \\
\hline APOV & $3.70 \mathrm{abcd}$ & 1.60 & 2.90 & 1.00 & 0.50 & 4.70 & 4.90 & 3.40 & 2.20 \\
\hline FEDECADE & $2.30 \mathrm{a}$ & 2.20 & 2.90 & 1.50 & 1.50 & 3.40 & 4.00 & 3.30 & 3.50 \\
\hline San Carlos & $4.50 \mathrm{~cd}$ & 1.90 & 4.10 & 2.80 & 0.50 & 2.00 & 2.60 & 2.00 & 0.50 \\
\hline Miss Ecuador & $4.60 \mathrm{~d}$ & 4.20 & 3.50 & 2.20 & 1.20 & 3.80 & 3.20 & 4.00 & 1.50 \\
\hline CORAGRICACE & $3.10 \mathrm{abc}$ & 1.70 & 2.80 & 2.20 & 1.30 & 3.20 & 1.10 & 2.30 & 2.30 \\
\hline Promedios & 3.50 & 1.80 & 3.10 & 1.90 & 1.00 & 3.30 & 3.00 & 3.20 & 1.70 \\
\hline
\end{tabular}

abcd Los promedios con letras distintas son estadísticamente diferentes (Kruskal y Wallis; $\mathrm{p}=0.05$ )

2004; Rodrigues et al., 2015).

Sabores básicos como la acidez y la astringencia, son menos familiares que el del cacao, influyendo sobre la precisión con que se cuantifica la intensidad sensorial de ambos; con frecuencia su descripción es más compleja (Bonvehi y Coll, 2000; Luna et al., 2002). Las mediciones y descripción de los marcadores sensoriales que se expresan con distintas intensidades en los cacaos finos de aroma, es decir las notas floral, frutal, nuez y caramelo, resulta algo más compleja y subjetiva, lo que dificulta la diferenciación y la selección de cultivares por atributos particulares de sabor en los procesos de mejoramiento genético (Eskes et al., 2007), así como en la diferenciación de orígenes comerciales. Incluso si estas sensaciones ya forman parte de nuestra biblioteca mental, como resultado de experiencias anteriores con otros alimentos, incluso con el mismo cacao, son sensaciones menos cotidianas y más finas.

Las diferencias en los promedios de fermentación (Cuadro 3) parece que también nutrieron la variación de los resultados sensoriales. La presencia de granospizarra con un promedio de $18 \%$ en la muestra de UCOCS, $11 \%$ en la muestra de COCPE, y granos-violeta con $40 \%$ en la muestra de FEDECADE, con seguridad influyeron en la calidad de su expresión sensorial. La evidencia sobre el efecto que ejerce la fermentación 
Cuadro 5. Matriz de correlación entre variables sensoriales

\begin{tabular}{|c|c|c|c|c|c|c|c|c|c|}
\hline Sabores & Cacao & Floral & Frutal & Nuez & Caramelo & Amargor & Acidez & Astringencia & Verde \\
\hline Cacao & 1 & & & & & & & & \\
\hline Floral & 0.31 & 1 & & & & & & & \\
\hline Frutal & 0.37 & 0.33 & 1 & & & & & & \\
\hline Nuez & 0.59 & -0.13 & 0.12 & 1 & & & & & \\
\hline Caramelo & -0.41 & 0.39 & 0.41 & -0.24 & 1 & & & & \\
\hline Amargor & -0.12 & -0.09 & -0.73 & -0.14 & -0.41 & 1 & & & \\
\hline Acidez & -0.11 & 0.31 & 0.38 & -0.63 & 0.26 & -0.09 & 1 & & \\
\hline Astringencia & -0.14 & 0.10 & -0.77 & -0.26 & -0.29 & 0.75 & 0 & 1 & \\
\hline Verde & -0.59 & -0.05 & -0.78 & -0.27 & 0.01 & 0.66 & -0.05 & 0.58 & 1 \\
\hline
\end{tabular}

En negrita, valores significativos $=0.05$

insuficiente de los granos sobre la expresión del sabor del cacao y demás rasgos sensoriales es amplia (Saltos, 2005; Amores et al., 2007, Jiménez et al., 2011).

El primer componente principal contiene el $35.20 \%$ de la varianza de la matriz de variables originales, mientras que el segundo componente principal contiene el 26.10\%; ambos suman 61.30\% (Cuadro 6). En el Cuadro 7, se observa que las variables originales con más aportación al primer componente principal son: frutal, verde y amargor, en el mismo orden. Las variables que más aportaron al segundo Componente principal son: acidez, floral y nuez.

Los coeficientes de correlación negativa, aunque bajos, entre el sabor a cacao con el amargor, acidez y astringencia, sugieren la presencia de una relación inversa en la expresión de estas variables. Pero también sugieren que son variables relativamente independientes en la dinámica de sus cambios, una aparente contradicción (Sandhya et al., 2016; Viogt et al., 2016). Este resultado amerita estudios adicionales para su clarificación por la relevancia que podría tener en la predicción de la calidad sensorial de granos de distintas variedades de cacao, al menos con relación a las características señaladas.

A pesar del insuficiente control de factores ajenos al experimento, aun fue posible observar a través del análisis de componentes principales, cierta estructuración de los puntos con la información de cada muestra. Según esta estructuración se conformaron dos grupos en el plano definido por los dos componentes principales con el mayor contenido de varianza. El patrón observado sugiere que las muestras tienden a juntarse a partir de semejanzas sensoriales que en conjunto las apartan del otro grupo cuyos miembros comparten otras características comunes. La investigación sobre diferenciación sensorial del cacao de distintas variedades y orígenes comerciales es más bien una actividad reciente. Al ser un ámbito científico muy joven se complica la interpretación de los resultados de estudios de este tipo por las amplias lagunas del marco teórico relevante al tema.

La distribución de los puntos de todas las muestras en el plano definido por los dos primeros componentes principales, permite observar que las muestras COCPE, UCOCS y APOV se agrupan por la presencia de los perfiles de amargor, astringencia y verde. FEDECADE y Buena Suerte tienen considerable intensidad de acidez, sin embargo se observa matices interesantes de notas especiales como dulce y floral. Fortaleza del Valle y Miss Ecuador poseen intensidades balanceadas de floral y frutal. Por otro lado, se agrupan Eloy Alfaro y San Carlos diferenciándose por la presencia del perfil frutal, cacao

Cuadro 6. Contribución (\%) de cada componente principal a la varianza acumulada de la nueva matriz de variables ortogonales

\begin{tabular}{|c|c|c|c|c|c|c|c|c|c|}
\hline & $\mathrm{F} 1$ & F2 & F3 & $\mathrm{F} 4$ & F5 & F6 & F7 & $\mathrm{F} 8$ & F9 \\
\hline Valor propio & 3.52 & 2.61 & 1.97 & 0.98 & 0.43 & 0.24 & 0.20 & 0.05 & 0.01 \\
\hline Varianza (\%) & 35.22 & 26.11 & 19.74 & 9.75 & 4.27 & 2.35 & 1.97 & 0.51 & 0.07 \\
\hline Acumulado (\%) & 35.22 & 61.33 & 81.07 & 90.83 & 95.1 & 97.45 & 99.41 & 99.93 & 100.00 \\
\hline
\end{tabular}

Número de valores propios triviales suprimidos: 1 
Cuadro 7. Contribución (\%) de las variables originales a la varianza total de cada uno de los componentes principales del APC

\begin{tabular}{lrrrrrrrrr}
\hline Características & \multicolumn{1}{c}{ F1 } & \multicolumn{1}{c}{ F2 } & \multicolumn{1}{c}{ F3 } & \multicolumn{1}{c}{ F4 } & \multicolumn{1}{c}{ F5 } & \multicolumn{1}{c}{ F6 } & \multicolumn{1}{c}{ F7 } & \multicolumn{1}{c}{ F8 } & \multicolumn{1}{c}{ F9 } \\
\hline Cacao & 5.65 & 0.90 & 37.26 & 1.63 & 0.53 & 0.25 & 0.07 & 44.28 & 5.62 \\
Floral & 0.71 & 21.18 & 9.98 & 10.65 & 17.39 & 18.38 & 0.12 & 5.44 & 9.78 \\
Frutal & 24.28 & 3.68 & 0.01 & 0.60 & 4.10 & 0.03 & 6.68 & 18.52 & 35.70 \\
Nuez & 2.95 & 13.75 & 13.34 & 16.67 & 16.65 & 10.66 & 5.33 & 7.31 & 8.57 \\
Caramelo & 2.16 & 13.01 & 10.77 & 31.89 & 0.10 & 15.95 & 6.87 & 17.90 & 0.02 \\
Amargor & 21.19 & 0.01 & 4.96 & 0.75 & 9.02 & 0.73 & 54.77 & 0.02 & 2.82 \\
Acidez & 0.21 & 24.42 & 0.57 & 27.79 & 10.52 & 3.88 & 8.91 & 0.58 & 10.96 \\
Astringencia & 20.51 & 0.81 & 4.27 & 0.20 & 19.54 & 34.79 & 1.18 & 4.29 & 9.22 \\
Verde & 21.19 & 0.64 & 1.33 & 9.09 & 13.09 & 14.09 & 15.92 & 1.29 & 16.13 \\
\hline
\end{tabular}

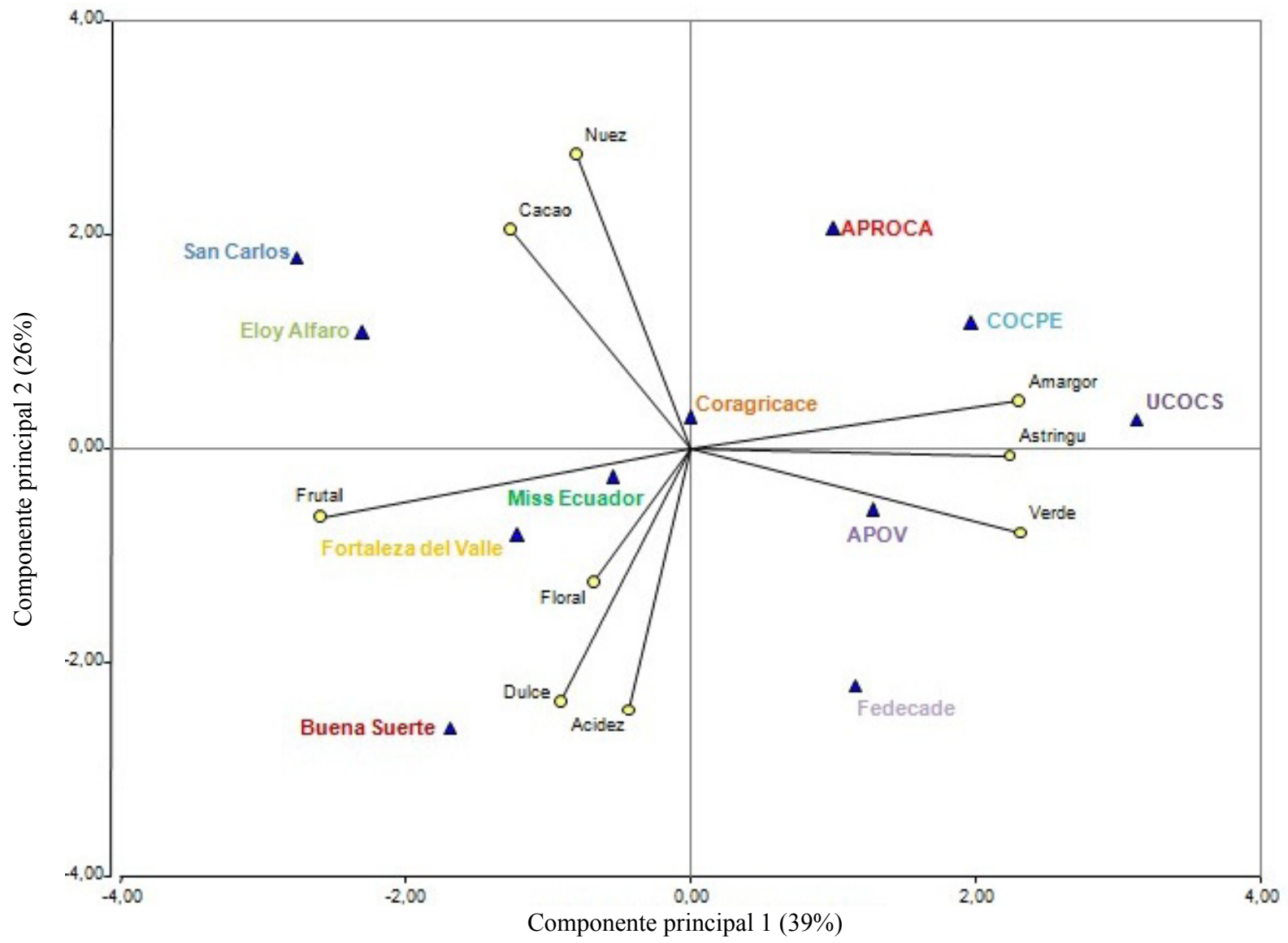

Figura 4. Distribución en el plano definido por los componentes principales 1 y 2 de los puntos con la información sensorial de las muestras de las distintas zonas cacaotera

y nuez. CORAGRICACE presenta combinaciones de cacao, floral, frutal y nuez, con bajos niveles de intensidad (Figura 4). APROCA se destaca por la presencia del sabor a nuez y cacao.

La gran cantidad de varianza acumulada en los dos primeros componentes principales, $39 \%$ y $26 \%$, respectivamente, confirman que ciertas variables intervinientes tienen una dinámica estrechamente asociadas entre ellas, y que en ciertos casos la asociación dio lugar a altos coeficientes de correlación; por tanto una mayor dependencia mutua. Esto demuestra que el análisis de componentes principales fue efectivo al 
concentrar en ambos componentes la mayor proporción de la variabilidad aportada por las 10 variables originales, recortando la información redundante de variables con alta correlación.

La aportación de la mayor cantidad de varianza por parte de las variables frutal, amargor y verde, sugiere que la insuficiente fermentación de ciertas muestras, se convirtió en una fuente de variación importante, al aumentar la intensidad de la expresión de características poco deseables, tal como ha sido revelado por varios estudios (Palacios, 2008; Jiménez et al., 2011; Solórzano, 2011). La importante aportación de las variables acidez, floral y nuez, sugiere que es posible revelar diferencias para estas características entre distintas zonas, con una lectura más precisa de los resultados sensoriales entre muestras dentro de la misma zona. El uso de al menos cinco repeticiones, así como un mejor control de la fermentación y tiempo de muestreo, son acciones que se pueden traducir en una menor variación de las lecturas para incrementar la precisión de los resultados en nuevos trabajos sobre el tema. Después de todo algunas diferencias ya han sido reportadas por actores del segmento industrial de la cadena (local e internacional) del cacao y chocolate (Ruiz et al., 2004; Cedeño, 2010). Hay que seguir trabajando en su comprobación empírica para confirmar que dichas diferencias realmente existen. De esta forma, será posible proporcionar un sustento científico al proceso de construcción de identidades con base al perfil de sabor para cacaos originarios de distintas zonas del país. La identidad sensorial bien definida puede convertirse en un factor clave de agregación de valor para cualquier origen local de cacao (Amores et al., 2009; Cedeño, 2010).

\section{Conclusiones}

$\mathrm{T}$ odas las muestras de cacao respondieron con perfiles sensoriales diferentes frente a la evaluación, aunque solo para una variable de sabor surgieron diferencias estadísticas entre ellos. La intensidad del sabor a cacao permitió separar estadísticamente las muestras analizadas, destacándose aquellas provenientes de las Asociaciones Miss Ecuador y APROCA por su mayor valoración para este marcador. La muestra correspondiente a Miss Ecuador presentó expresiones más intensas, no solo para el sabor a cacao, sino también las notas sensoriales asociadas a los cacaos finos o de aroma, particularmente floral y frutal. Se encontró una correlación inversa entre el sabor a cacao y las notas sensoriales asociadas a los cacaos finos, con el amargor, acidez astringencia. Estas diferencia reales contribuyen a la identificación de perfiles sensoriales propios de cada una de las zonas en estudio.

\section{Bibliografía}

Amores, F., Butter, D., Ramos, G., Sukha, D., Espin, S., Gómez, A., Zambrano, A., Jiménez, J., Hollywood, N., Van Loo, R., and Seguine, E. 2007. Reporte final. Project to establish the physical, chemical and organoleptic parameters to differentiate fine a flavor and bulk cacao. INIAP/CFC/ICCO project. p.84.

Amores, F., Palacios, Á., Jiménez, J., Zhang, D. 2009. Entorno ambiental, genético, atributos de calidad y singularización del cacao en el nor oriente de la provincia del Ecuador. Extensión Experimental Tropical Pichilingue. INIAP, SENACYT, USDA. Boletín Técnico \# 135. Quevedo, Ecuador. p.99.

Ardhana, M., Fleet, G. 2003. The microbial ecology of cocoa bean fermentation in Indonesia. International Jornal of Food Microbiology 86: 87-99.

Barel, M., Leon, D., Vincent, J. 1985. Influence of cocoa fermentation time on the production of pyrazines in chocolate. Café Cacao the 29(4): 277-286.

Bonvehi, J., Coll, F. 2000. Evaluation of purine alkaloids and diketopiperazines contents in processed cocoa powder. European Food Research and Technology 210: $189-195$

Briz, J., García, R. 2004. Análisis sensorial de productos alimentarios metodología y aplicación a casos prácticos. 2da edición. Madrid- España

Cedeño, P. 2010. Determinación de perfiles organolépticos en ocho grupos de cacao mediante la degustación de licor de cacao y chocolates oscuros elaborados artesanalmente. Tesis Ing. Agroindustrial. Escuela Superior Politécnica Agropecuaria de Manabí Manuel Félix López. Manabí Ecuador.

Charlton, A., Baxter, N., Khan, M., Moir, A., Haslam, E., Davies, A., Williamson, M. 2002. Polyphenol/ peptide binding and precipitation. Journal of Agricultural and Food Chemistry 50(6): 15931601.

Cros, E. 2004. Factores que afectan el desarrollo del sabor a cacao bases bioquímicas del perfil aromático. Memoria. Taller Internacional calidad Integral del cacao: Teoría y Práctica (2004) INIAP / EET-P Quevedo, Ecuador.

Eskes, A.B, Guarda, D., Garda, L.F., Garcia, P.V. 2007. Is genetic variation for sensory trants of cocoa pulp related to fine flavor cocoa trants. INGENIC Newslette 11: 22-28.

Fowler, M. 2009. Cocoa beans: from tree to factory. In S.T. Beckett (Ed.) Industrial chocolate manufacture and use. Chichester: Blackwell Publishing Ltd. p $10-47$. 
Ho, V., Zhao, J., Fleet, G. 2015. The effecto of lactic acid bacteria on cocoa beam fermentation. International Journal of food microbiology 205: 54-67

Instituto Ecuatoriano de Normalización. 2006. Norma Técnica Ecuatoriana NTE INEN 176. Cuarta Revisión.

Instituto Nacional de Meteorología e Hidrología (INAMHI). 2015. Anuario meteorológico $\mathrm{N}^{\circ}$ 522012.

International Organization for Standardization (ISO). 1988. Sensory analysis. General guidance for the design of test rooms. ISO 8589: 1988.

Jiménez, J., Amores, F., Nicklin, C., Rodríguez, D., Zambrano, F., Reynel, V., Dueñas, A., Cedeño, P. 2011. Micro fermentación y análisis sensorial para la selección de árboles superiores de cacao. Boletín técnico No 140. INIAP/ EET-P, Quevedo, Ecuador. p. 48.

Loor, R. 2002. Caracterización morfológica y molecular de 37 clones de cacao (Theobroma cacao L.) Nacional de Ecuador. Colegio de Posgraduacion, Instituto de Enseñanza e Investigación en Ciencias Agrícolas; Instituto de Recursos Genéticos y Productividad; programa de Genética, Maestro en Ciencias. p 52

Luna, F., Crouzillat, D., Cirou, L., Bucheli, P. 2002. Chemical composition and flavor of Ecuadorian cocoa liquor. Journal of Agricultural and Food Chemistry 50(12): 3527-3532.

Oracz, J., Nebesny, E. 2014. Influence of roasting conditions on the biogenic amine content in cocoa beans of different Theobroma cacao cultivars. Food Research International 55: 1-10

Palacios, A. 2008. Establecimiento de parámetros (físicos, químicos, y organolépticos) para diferenciar y valorizar el cacao (Theobroma cacao L.) producido en dos zonas identificadas al norte y sur del litoral ecuatoriano. Tesis Ing. Agr. Universidad Técnica de Manabí- Ecuador. 189 p.

Pérez, R. 2009. La calidad del cacao. Memoria programa de capacitación a facilitadores y agricultores en la cadena del cacao. Quito- Ecuador. p. 44-45.

Puerari, C., Magalhaes, K., Schwan, R. 2012. New cacao pulp-based kéfir beverages: Microbiologica chemical composition and sensory analysis. Food Research Intenational 48(2): 634-640.

Ribas, A., Gratacós, M., Sárraga, C., Guárdia, D., García, J., Castellari, M. 2014. Stability of phenolic compunds in dry fermented sausages added with cocoa and grope seed extracts. LWT - Food Science and Technology 57(1): 329-336.
Rodrigues, J., Condino, J., Pinheiro, A., Nunes, C. 2015. Temporal dominaceof sensations of chocolate bars with different cocoa contents: Multivariate approaches to assess TDS profile. Food Quality and Preference 47: 91-96

Ruiz, M., Mera, O., Prado, A., Cedeño, W. 2004 Influencia de la época de cosecha en la calidad del licor de cacao tipo Nacional. Espamciencia 5(2): 73-85.

Sacchetti, G., Ioannone, F., Gregorio, M., Di, C. 2015. Non enzymatic browning during cocoa roasting as affected by processing time and temperature. Journal of Food Engineering 169: 4-52.

Saltos, A. 2005. Efecto de métodos de fermentación, frecuencias de remoción y volúmenes variables de masa fresca de cacao sobre la calidad física y organoléptica del "Complejo Nacional x Trinitario". Tesis Ing. Agr. Universidad de Guayaquil, Vinces Ecuador. $5 \mathrm{p}$.

Sandhya, M., Yallappa, B., Varadaraj, M., Puranaik, J., Rao, L., Janardhan, P., Murthy, P. 2016. Inoculum of the starter consortia and interative metabolic process in enhancing quality of cocoa bean (Theobroma cacao) fermentation. LWT - Food Science and Technology 65: 731-738.

Solórzano, E. 2011. Evaluación sensorial del cacao Nacional fino o de aroma procedente de diferentes zonas del país. Tesis Ing. Agroindustrial. Universidad Técnica Estatal de Quevedo. Quevedo, Ecuador.

Sukha, D., Butler, D. 2006. The CFC/ICCO/INIAP Cocoa Flavour Project - Investigating the spectrum of fine flavour within genotypes and between origins, INGENINC Newsletter 10: 22-25

Tran, P., Van de Walle, D., De Clercq, N., De Winne, A., Kadow, D., Lieberei, R., Messens, K., Tran, D., Dewettinck, K., Durme, J. 2015. Assessing cocoa aroma quality by multiple analytical approaches. Food Research International.

Voigt, J., Janek, K., Textoris-taube, K., Niewienda, A., Wostemeyer, J. 2016. Partial purification and characterization of the peptide precursors of the cocoa-specific aroma components. Food Chemistry 192: 706-713

Zyzelewicz, D. Budryn, G., Krysiak, W., Oracz, J., Nebesny, E., Bojczuk, M. 2014. Influence of roasting conditions on fatty acid composition and oxidative changes of cocoa butter extracted from cocoa beam of Forastero variety cultivaded in Togo. Food Research International. 63: 328-343. 